\title{
Inelastic and elastic collision rates for triplet states of ultracold strontium
}

\author{
A. Traverso, R. Chakraborty, Y. N. Martinez de Escobar, P. G. Mickelson, S. B. Nagel, M. Yan, and T. C. Killian \\ Department of Physics and Astronomy, Rice University, Houston, Texas 77251, USA
}

(Received 4 September 2008; revised manuscript received 13 May 2009; published 5 June 2009)

\begin{abstract}
We report measurement of the inelastic and elastic collision rates for ${ }^{88} \mathrm{Sr}$ atoms in the $(5 s 5 p){ }^{3} P_{0}$ state in a crossed-beam optical dipole trap. Since the $(5 s 5 p)^{3} P_{0}$ state is the lowest level of the triplet manifold, large loss rates indicate the importance of principle-quantum-number-changing collisions at short range. We also provide an estimate of the collisional loss rates for the $(5 s 5 p)^{3} P_{2}$ state. Large loss-rate coefficients for both states indicate that evaporative cooling toward quantum degeneracy in these systems is unlikely to be successful.
\end{abstract}

DOI: 10.1103/PhysRevA.79.060702

PACS number(s): $34.50 . \mathrm{Cx}$

Metastable ${ }^{3} P_{J}$ states of alkaline-earth-metal atoms and atoms with similar electronic structure (Fig. 1) display vastly different optical and ultracold collisional properties compared to states found in alkali-metal atoms more commonly used in ultracold atomic physics experiments. The extremely long-lived ${ }^{3} P_{0}$ states in $\mathrm{Sr}$ and $\mathrm{Yb}$ serve as the upper levels in neutral-atom optical frequency standards [1]. The weakly allowed ${ }^{1} S_{0}-{ }^{3} P_{1}$ intercombination transition serves as the basis for powerful laser-cooling techniques [2] and may enable useful optical tuning of the ground-state scattering length [3]. ${ }^{3} P_{2}$ atoms interact through long-range anisotropic interactions $[4,5]$ that allow magnetic tuning of the interactions and cause rapid inelastic collisional losses [6-8]. ${ }^{3} P_{J}$ states of alkaline-earth-metal atoms have also been proposed for lattice-based quantum computing $[9,10]$.

Here, we report measurement of the inelastic and elastic collision rates for ${ }^{88} \mathrm{Sr}$ atoms in the $(5 s 5 p)^{3} P_{0}$ state in a crossed-beam optical dipole trap. The measurement of the ultracold collisional properties of the ${ }^{3} P_{0}$ state is of great interest because of its role in optical clocks. We also report an estimate of the collisional loss rates for the ${ }^{88} \mathrm{Sr}(5 s 5 p)^{3} P_{2}$ state. Large loss-rate coefficients for both states make efficient evaporative cooling in the ultracold regime unlikely.

Early laser-cooling experiments with $\mathrm{Sr}$ [11] and $\mathrm{Ca}$ [12] showed that it is straightforward to magnetically trap metastable ${ }^{3} P_{2}$ atoms through natural decay in a magneto-optical trap. This generated interest in the possibility of achieving quantum degeneracy in this state and motivated calculations that found novel collisional properties of the metastable ${ }^{3} P_{J}$ levels [4]. Magnetic dipole-dipole and electric quadrupolequadrupole interactions between ${ }^{3} P_{2}$ atoms produce anisotropic long-range potentials with bound states and collisional rates that can be tuned with magnetic field. Reference [5] showed that $s$-wave colliding states can be coupled to much higher partial waves of outgoing channels of other magnetic sublevels even if the initial state is maximally spin polarized. This leads to two-body inelastic loss rates in magnetically trapped samples [6] that are on the order of elastic collision rates, making efficient evaporative cooling toward quantum degeneracy of ${ }^{3} P_{2}$ atoms in a magnetic trap unlikely. This was confirmed in experiments with magnetically trapped $\mathrm{Ca}$ [7].

Theory [4-6] only considered Zeeman-sublevel-changing (ZSLC) collisions mediated by long-range interactions, which led to speculation $[6,7]$ that losses in optical traps might be low enough to allow evaporation. However, large loss rates were also found in gases of ${ }^{3} P_{2} \mathrm{Yb}$ atoms held in an optical dipole trap, which suggested that fine-structurechanging (FSC) collisions at short range are also significant [8].

By studying ultracold collisions between ${ }^{3} P_{0}$ atoms, which occupy the lowest level of the fine-structure triplet, we remove the possibility of ZSLC and FSC collisions and probe the importance of principle-quantum-number-changing (PQNC) collisions. For ${ }^{3} P_{0}$ atoms, a PQNC collision results in one atom in the ground state, which is referred to as an energy-pooling collision [13]. Differences between ${ }^{3} P_{0}$ and ${ }^{3} P_{2}$ collisions may also arise because ${ }^{3} P_{0}$ atoms are isotropic and lack magnetic-dipole and electric-quadrupole moments. ${ }^{88} \mathrm{Sr}$ has nuclear spin $I=0$.

At higher temperatures, de-excitation of $\operatorname{Sr}(5 s 5 p)^{3} P_{J}$ states due to collisions with background noble-gas atoms $[14,15]$ and ground state $\mathrm{Sr}[16]$ has been well studied. Energy-pooling $(5 s 5 p)^{3} P_{J}+(5 s 5 p)^{3} P_{J^{\prime}} \rightarrow\left(5 s^{2}\right)^{1} S$ $+(5 s 6 s)^{3,1} S$ collision rates have also been measured in a $\mathrm{Sr}$ heat pipe [13]. All of these studies, as well as ultracold experiments with ${ }^{3} P_{2}$ levels in other atoms $[7,8]$, worked in a regime where fine-structure changing collisions were energetically allowed.

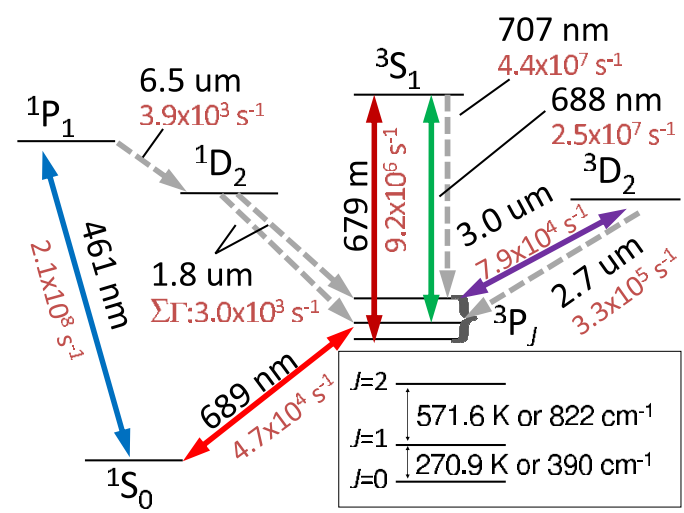

FIG. 1. (Color online) Strontium atomic levels. Decay rates $\left(\mathrm{s}^{-1}\right)$ and excitation wavelengths are given for selected transitions. Laser light used for the experiment is indicated by solid lines. Dashed lines indicate transitions that are only made due to spontaneous decay. All levels with energy below $43000 \mathrm{~K}$ are shown. The energy of the ${ }^{3} S_{1}$ state is $41780.5 \mathrm{~K}$. The inset gives the energy splittings of the metastable ${ }^{3} P_{J}$ levels. 


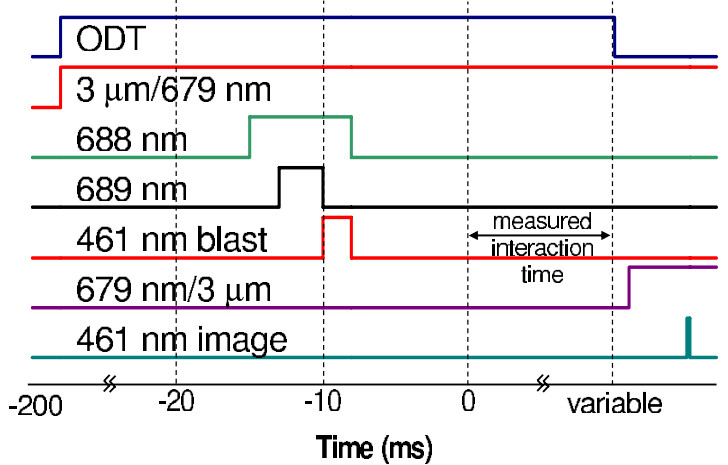

FIG. 2. (Color online) Timing diagram for measuring metastable collision dynamics. Note the breaks in the time axis. Simultaneous application of the 688 and $689 \mathrm{~nm}$ lasers populates the metastable triplet levels. A strong $461 \mathrm{~nm}$ beam applied after the initial optical pumping ensures that no atoms remain in the ${ }^{1} S_{0}$ state. For measuring ${ }^{3} P_{0}$ dynamics and to keep the ${ }^{3} P_{2}$ level unpopulated, the $3 \mu \mathrm{m}$ laser is on the entire time, and the $679 \mathrm{~nm}$ is pulsed on to repump the ${ }^{3} P_{0}$ atoms to the ground state. For measuring ${ }^{3} P_{2}$ dynamics, the timing of these two lasers is interchanged as indicated in the timing labeling.

Laser-cooling and trapping aspects of the experiment are described in $[11,17]$. To obtain higher density and longer sample lifetime, and to trap all electronic states, atoms are transferred to an optical dipole trap (ODT) of controllable trap depth as described in [17], yielding equilibrium temperatures of between of 3 and $15 \mu \mathrm{K}$ and peak densities up to $10^{14} \mathrm{~cm}^{-3}$. The potential seen by the atoms is determined from measured ODT laser properties and well-known atom polarizabilities $[1,18]$ and measured trap oscillation frequencies [19].

We then pump atoms via ${ }^{1} S_{0} \rightarrow{ }^{3} P_{1} \rightarrow{ }^{3} S_{1}$ followed by natural decay to the metastable state of interest by applying the $689 \mathrm{~nm}$ laser for $3 \mathrm{~ms}$ while the $688 \mathrm{~nm}$ laser is on (Fig. 2 ). Atoms decaying from the ${ }^{3} S_{1}$ state to the wrong metastable state are repumped with a clean-up laser at $3.0 \mu \mathrm{m}$ for experiments with ${ }^{3} P_{0}$ atoms and with a $679 \mathrm{~nm}$ laser for experiments with ${ }^{3} P_{2}$ atoms. The clean-up repumper is turned on during the loading stage of the ODT and left on for the rest of the experiment. Any atoms remaining in the ground state are removed from the trap with a $2 \mathrm{~ms} 461 \mathrm{~nm}$ pulse. We typically obtain $10^{6}$ metastable atoms at a temperature near $10 \mu \mathrm{K}$, a density as high as $10^{13} \mathrm{~cm}^{-3}$, and phase-space density as high as $10^{-3}$.

The zero of time for interaction studies is set after the metastable atoms have equilibrated for approximately $10 \mathrm{~ms}$ after the $689 \mathrm{~nm}$ laser is extinguished. The end of the interaction time is determined by the extinction of the ODT, after which the atoms ballistically expand and fall under the influence of gravity. The density drops rapidly enough that atomatom interactions cease on a millisecond time scale.

The number of atoms and sample temperature are determined with time-of-flight absorption imaging using the ${ }^{1} S_{0}$ $-{ }^{1} P_{1}$ transition. This necessitates repumping the atoms to the ground state during the ballistic expansion by applying the $679 \mathrm{~nm}$ laser in the case of ${ }^{3} P_{0}$ atoms and $3.0 \mu \mathrm{m}$ laser in the case of ${ }^{3} P_{2}$ atoms. It is important to release the ODT before repumping because the repump lasers cause densitydependent light-assisted losses. Complete repumping requires a few milliseconds.

Photon recoil during optical pumping to the metastable state before the interaction time and then to the ground state for imaging affects the atomic momentum distribution, which complicates measurement of the temperature. The recoil energy for a red photon is $\hbar^{2} k^{2} /\left(m k_{B}\right)=0.5 \mu \mathrm{K}$, and from the branching ratios shown in Fig. 1, each atom is expected to scatter about five red photons during initial excitation and one to three photons during repumping. The repump lasers are all aligned horizontally, so we expect several $\mu \mathrm{K}$ of extra heating along this axis. Recoil from the $3 \mu \mathrm{m}$ photons is insignificant.

An approximate description of the loss of atoms during the interaction time can be derived from a local equation for the evolution of the atomic density, $\dot{n}=-\beta n^{2}-\Gamma n$, which, assuming constant sample temperature, can be integrated spatially to yield the evolution in atom number

$$
N(t)=\frac{N_{0} e^{-\Gamma \mathrm{t}}}{1+\frac{N_{0} \beta V_{2}}{\Gamma V_{1}^{2}}\left(1-e^{-\Gamma \mathrm{t}}\right)} .
$$

$N_{0}$ is the number at the beginning of the interaction time, and the one-body loss rate, $\Gamma$ is due to background collisions. The effective volumes, indexed by subscript $q$, are defined by

$$
V_{q}(T)=\int d^{3} r\left[n(\mathbf{r}) / n_{0}\right]^{q},
$$

where $n(\mathbf{r})$ is the spatial density distribution calculated from the trap potential and atom temperature including effects due to truncation of the Boltzmann distribution [20], and $n_{0}$ is the peak density in the trap. The spatial integral extends over the region contained in the trap.

Figure 3 shows representative data for temperature and number evolution for atoms in the ${ }^{3} P_{0}$ state. The horizontal axis $(x)$ is about $2 \mu \mathrm{K}$ hotter than the vertical, which we attribute to photon recoil heating during optical pumping. The temperature change during the interaction time and the $x-y$ temperature difference are both small, so an assumption of constant temperature is a reasonable approximation, and Eq. (1) fits the data well. The sample temperature used to calculate effective volumes is taken as the average of $T_{x}$ and $T_{y}$. A fit neglecting two-body decay reproduces the data poorly, confirming the importance of two-body effects.

Values of $\beta$ derived from a series of decay curves are shown in Fig. 4. The dominant uncertainties in $\beta$ are systematic and reflected in the error bars. Uncertainty in temperature of $2 \mu \mathrm{K}$ only contributes a $10 \%$ uncertainty in $\beta$, while uncertainty in the trap oscillation frequencies at the $20 \%$ level causes a $60 \%$ uncertainty. At our level of uncertainty, we observe no temperature dependence and find $\beta$ $=(2.3 \pm 1.4) \times 10^{-17} \mathrm{~m}^{3} / \mathrm{s}$.

As pointed out in [21], both inelastic-collisional loss and evaporation due to elastic collisions are two-body loss processes contributing to $\beta=\beta_{i n}+f \beta_{e}$, where $\beta_{\text {in }}$ is the inelastic- 

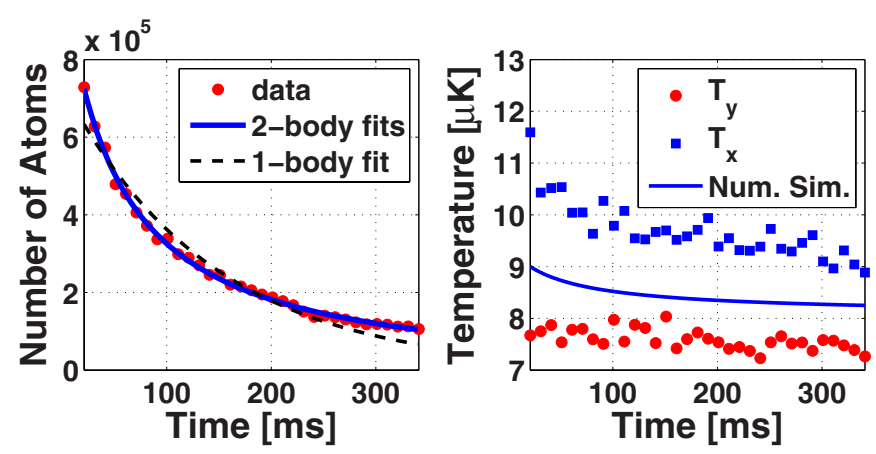

FIG. 3. (Color online) Left: number of trapped atoms as a function of time for studies of the ${ }^{3} P_{0}$ state. Two-body fits allowing for one- and two-body losses using Eq. (1) and the numerical simulation described in the text are identical. The statistical uncertainties in the two-body loss-rate constant, $\beta=18 \pm 3 \times 10^{-18} \mathrm{~m}^{3} / \mathrm{s}$, and one-body loss rate, $\Gamma=1.4 \pm 0.9 \mathrm{~s}^{-1}$, are dominated by correlation between the parameters, but $\beta$ is determined much better than systematic uncertainties described in the text and is the dominant loss mechanism. The one-body fit sets $\beta=0$ and is not a good description of the data. The observed value for $\Gamma$ is consistent with the decay rate for ${ }^{1} S_{0}$ atoms, which is dominated by background gas collisions. Right: temperature evolution of the trapped sample. The fit is from the numerical simulation. The ODT trap depth resulting from symmetric saddle points located approximately along the horizontal axes $\left(x\right.$ and $z$ ) is $U_{\text {trap }} / k_{B}=20 \mu \mathrm{K}$. The potential barrier for escape along the vertical $(y)$ direction is $30 \mu \mathrm{K}$.

collision-loss rate constant, $\beta_{e l}$ is the elastic-collision rate constant, and $f$ is the fraction of elastic collisions resulting in an evaporated atom. The rate constants can be expressed as $\beta_{\text {in }}=\beta /(1+f \gamma)$ and $\beta_{e l}=\gamma \beta /(1+f \gamma)$, where the ratio of rate constants is

$$
\gamma \equiv \frac{\beta_{e l}}{\beta_{i n}}=\frac{\bar{E}-\bar{E}_{i n}}{f\left(\bar{E}_{e l}-\bar{E}\right)} .
$$

$\bar{E}$ is the average energy of the trapped atoms, $\bar{E}_{i n}$ is the average energy of atoms lost due to inelastic collisions, and $\bar{E}_{e l}$ is the average energy of atoms lost due to evaporation. Parameters on the right-hand side of Eq. (3) can be calculated from the trapping potential and equilibrium atom temperature. In our case, these quantities must be evaluated numerically because the potential is not amenable to analytic solutions and truncation of the Boltzmann distribution is significant [20]. For the various conditions studied here the ranges of parameters are $0.07<f<0.3$ and $0.6<\gamma<3.7$, and we obtain $\beta_{i n}=(1.9 \pm 1.2) \times 10^{-17} \mathrm{~m}^{3} / \mathrm{s}$ (Fig. 4). The data suggest that $\beta_{e l}$ increases slightly with temperature, as one would expect since $\beta_{e l}=\sigma \bar{v} \sqrt{2}$, where $\sigma$ is the elastic scattering cross section and $\bar{v}$ is the mean thermal velocity. The error bars in these measurements are dominated by the trap uncertainties. Our treatment assumes ergodicity, which is reasonable given the small difference between $T_{x}$ and $T_{y}$.

To check that the assumption of an equilibrium temperature does not introduce significant bias, we adapted a numerical model of the dynamics of atoms in an ODT [22] designed for a system with high $\eta \equiv U_{\text {trap }} / k_{B} T$ to our low- $\eta$
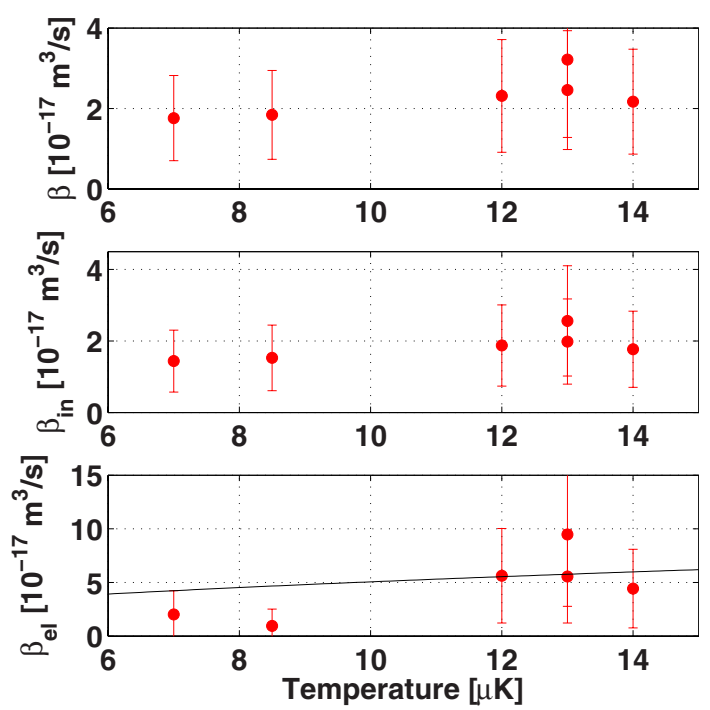

FIG. 4. (Color online) Two-body decay rate measurements for the ${ }^{3} P_{0}$ state. The solid line is $\beta_{e l}=\sigma \bar{v} \sqrt{2}$, where $\sigma=8 \pi a^{2},|a|$ $=100 a_{0}$, and $\bar{v}=\sqrt{8 k_{B} T / \pi m}$.

situation by incorporating the evaporation treatment of [20] and numerically calculating all effective volumes and related integrals using the actual trap shape and a truncated momentum distribution. The details of the model are given in [23]. Fits to both number and temperature evolution (Fig. 3) yielded the same values of $\beta_{i n}$ and $\beta_{e l}$ as above. Assuming pure $s$-wave elastic collisions, this simulation also allows us to provide an estimate of the magnitude of the ${ }^{3} P_{0}$ scattering length $|a|=100 \pm 50 a_{0}$.

Measurement of the number and temperature evolution for atoms in the ${ }^{3} P_{2}$ state together with numerical simulations assuming an equal trap depth for all magnetic sublevels constrain $\beta_{\text {in }}=(1.3 \pm 0.7 \pm 0.7) \times 10^{-16} \mathrm{~m}^{3} / \mathrm{s}$ reasonably well. The first uncertainty comes from the fit and the second uncertainty is systematic from knowledge of the trap potential. Several factors that increase uncertainty in determination of $\beta$ in this case must be addressed. The temperature measurement along $x$ is distorted by a high density of atoms evaporating into the arms of the crossed-dipole trap. However, the numerical simulation and elastic-collision rate implied by the low $T_{y}$ indicate that the temperature change and lack of equilibrium are not significantly worse than in ${ }^{3} P_{0}$. The different magnetic sublevels have different ac Stark shifts and experience optical trap depths that vary by almost a factor of 2. This complicates modeling of the system and introduces ZSLC-collisional heating and loss processes. But this would only increase $\beta$, and we take the observed value as an upper limit for the rate constant for FSC and PQNC collisional loss in this state.

The $\beta_{i n}$ for $\mathrm{Sr}^{3} P_{0}$, which can only reflect PQNC collisions, is a factor of 2 larger than $\beta_{\text {in }}$ for optically trapped $\mathrm{Yb}^{3} P_{2}$ [8] and a factor of 5 less than in $\mathrm{Sr}^{3} P_{2}$. The ${ }^{3} P_{2}$ states are sensitive to PQNC and FSC processes. ZSLC collisional loss rates in magnetically trapped ${ }^{3} P_{2}$ are 1 order of magnitude greater [6], as found in magnetically trapped $\mathrm{Ca}[7]$. 
One expects higher PQNC collision rates for ${ }^{3} P_{2}$ collisions than for ${ }^{3} P_{0}$ collisions because the process ${ }^{3} P_{J}+{ }^{3} P_{J}$ $\rightarrow\left(5 s^{2}\right)^{1} S_{0}+(5 s 6 s)^{3} S_{1}+\Delta E$ is allowed for $J=2 \quad\left(\Delta E / k_{B}\right.$ $=1091 \mathrm{~K})$ and energetically suppressed for $J=0\left(\Delta E / k_{B}\right.$ $=-581 \mathrm{~K}$ ). (See Fig. 1.) In light of the observed values of $\beta_{\text {in }}$ this implies that PQNC collisions make a significant contribution to the inelastic collision rate for ultracold ${ }^{3} P_{2}$ states and as predicted [6], ZSLC collisions should be much more rapid.

In conclusion, this work has highlighted the important role of PQNC collisions in ultracold metastable triplet levels in two-valence-electron atoms. It has also provided a measurement of the inelastic and elastic collision properties for ultracold atoms in a ${ }^{3} P_{0}$ state and an estimate of collisional properties for ${ }^{3} P_{2} \mathrm{Sr}$ atoms. These levels are of significant current interest in applications such as atomic clocks and in fundamental studies in ultracold atomic physics. The techniques developed here demonstrate the production of a cold and dense sample of ${ }^{3} P_{0} \mathrm{Sr}$ atoms, which is a prerequisite for photoassociative spectroscopy of atoms in this state and may allow determination of dipole matrix elements and blackbody radiation shifts that limit the accuracy of optical clocks $[1,24]$.

This research was supported by the Welch Foundation (Grant No. C-1579), National Science Foundation (Grant No. PHY-0555639), and the Keck Foundation. We thank D. Comparat for sharing the numerical code for simulating evaporative cooling.
[1] J. Ye, H. J. Kimble, and H. Katori, Science 320, 1734 (2008).

[2] H. Katori, T. Ido, Y. Isoya, and M. Kuwata-Gonokami, Phys. Rev. Lett. 82, 1116 (1999).

[3] R. Ciurylo, E. Tiesinga, and P. S. Julienne, Phys. Rev. A 71, 030701(R) (2005).

[4] A. Derevianko, S. G. Porsev, S. Kotochigova, E. Tiesinga, and P. S. Julienne, Phys. Rev. Lett. 90, 063002 (2003).

[5] R. Santra and C. H. Greene, Phys. Rev. A 67, 062713 (2003).

[6] V. Kokoouline, R. Santra, and C. H. Greene, Phys. Rev. Lett. 90, 253201 (2003).

[7] D. Hansen and A. Hemmerich, Phys. Rev. Lett. 96, 073003 (2006).

[8] A. Yamaguchi, S. Uetake, D. Hashimoto, J. M. Doyle, and Y. Takahashi, Phys. Rev. Lett. 101, 233002 (2008).

[9] A. Derevianko and C. C. Cannon, Phys. Rev. A 70, 062319 (2004).

[10] A. J. Daley, M. M. Boyd, J. Ye, and P. Zoller, Phys. Rev. Lett. 101, 170504 (2008).

[11] S. B. Nagel et al., Phys. Rev. A 67, 011401(R) (2003).

[12] D. P. Hansen, J. R. Mohr, and A. Hemmerich, Phys. Rev. A 67, 021401(R) (2003).

[13] J. F. Kelly, M. Harris, and A. Gallagher, Phys. Rev. A 38,
1225 (1988).

[14] J. F. Kelly, M. Harris, and A. Gallagher, Phys. Rev. A 37, 2354 (1988).

[15] C. Redondo, M. N. Sanchez Rayo, P. Ecija, D. Husain, and F. Castano, Chem. Phys. Lett. 392, 116 (2004).

[16] C. Redondo, M. N. Sanchez Rayo, J. A. Fernandez, D. Husain, and F. Castano, Chem. Phys. 264, 123 (2001).

[17] Y. N. Martinez de Escobar et al., Phys. Rev. A 78, 062708 (2008).

[18] S. G. Porsev, A. D. Ludlow, M. M. Boyd, and J. Ye, Phys. Rev. A 78, 032508 (2008).

[19] S. Friebel, C. DAndrea, J. Walz, M. Weitz, and T. W. Hänsch, Phys. Rev. A 57, R20 (1998).

[20] O. J. Luiten, M. W. Reynolds, and J. T. M. Walraven, Phys. Rev. A 53, 381 (1996).

[21] R. deCarvalho and J. Doyle, Phys. Rev. A 70, 053409 (2004).

[22] D. Comparat, A. Fioretti, G. Stern, E. Dimova, B. Laburthe Tolra, and P. Pillet, Phys. Rev. A 73, 043410 (2006).

[23] M. Yan, R. Chakraborty, P. G. Mickelson, Y. N. M. de Escobar, and T. C. Killian, e-print arXiv:0905.2223.

[24] S. G. Porsev and A. Derevianko, Phys. Rev. A 74, 020502(R) (2006). 